\title{
Aromatic, aliphatic, and the unidentified 21 micron emission features in proto-planetary nebulae
}

\author{
Bruce J. Hrivnak ${ }^{1}$, Kevin Volk ${ }^{2}$, T. R. Geballe ${ }^{2}$, and Sun Kwok ${ }^{3}$ \\ ${ }^{1}$ Department of Physics \& Astronomy, Valparaiso University, \\ Valparaiso IN, 46383, USA \\ email: bruce.hrivnak@valpo.edu \\ ${ }^{2}$ Gemini Observatory, 670 North A'ohoku Place, Hilo, HI 96720, USA \\ email: kvolk@gemini.edu, tgeballe@gemini.edu \\ ${ }^{3}$ Department of Physics, University of Hong Kong, Hong Kong, China \\ email: sunkwok@hku.hk
}

\begin{abstract}
Aromatic features at 3.3, 6.2, 7.7, 8.6, $11.3 \mu \mathrm{m}$ are observed in proto-planetary nebulae (PPNe) as well as in PNe and $\mathrm{H}$ II regions. Aliphatic features at 3.4 and $6.9 \mu \mathrm{m}$ are also observed; however, these features are often stronger in PPNe than in PNe. These observations suggest an evolution in the features from simple molecules $\left(\mathrm{C}_{2} \mathrm{H}_{2}\right)$ in AGB stars to aliphatics in PPNe to aromatics in PNe. In the same carbon-rich PPNe, a strong, broad, unidentified $21 \mu \mathrm{m}$ emission feature has been found. We will present recent observations of the aromatic, aliphatic, and $21 \mu \mathrm{m}$ emission features, along with $\mathrm{C}_{2} \mathrm{H}_{2}(13.7 \mu \mathrm{m})$ and a new feature at $15.8 \mu \mathrm{m}$, and discuss correlations among them and other properties of these PPNe.
\end{abstract}

Keywords. Astrochemistry, circumstellar matter, ISM: lines and bands, infrared: ISM, stars: AGB and post-AGB, planetary nebulae: general

\section{Background and New Observations}

Aromatic hydrocarbon emission features at 3.3, 6.2, 7.7, 8.6, and $11.3 \mu \mathrm{m}$, often attributed to PAHs, are observed in the spectra of various objects with hot irradiating sources; planetary nebulae (PNe), H II regions, reflection nebulae. They have also been observed in proto-planetary nebulae $(\mathrm{PPNe})$, objects in the short-lived ( $1000 \mathrm{yr})$ transitional phase between AGB stars and PNe. In PPNe, the circumstellar envelope is detached but the central star is not hot enough to photo-ionize the nebula and is typically of spectral type F-G. Aliphatic emission features at 3.4 and $6.9 \mu \mathrm{m}$ are also seen in PPNe and are often stronger than in PNe (Geballe 1997, Geballe et al. 1992). This suggests an evolution in the carbon chemistry of the circumsteller envelopes from $\mathrm{C}_{2} \mathrm{H}_{2}$ to aliphatics to aromatics as C-rich stars evolve rapidly from AGB to PPN to PN phases (Kwok 2004).

The unidentified $21 \mu \mathrm{m}$ emission feature, first seen in IRAS spectra of four C-rich PPNe (Kwok et al. 1989), has subsequently been observed in additional C-rich PPNe with ISO (Volk et al. 1999) and recently with Spitzer. This $21 \mu \mathrm{m}$ feature has been detected only in C-rich objects and essentially only in PPNe (and perhaps weakly in a few AGBs and young PNe). Suggested identifications include PAHs, TiC, SiC (see Speck \& Hofmeister 2004 and references therein), and FeO (Guha Niyogi et al., these proceedings).

New $3 \mu \mathrm{m}$ spectra have been obtained of seven PPNe. All show the $3.3 \mu \mathrm{m}$ and most show the $3.4 \mu \mathrm{m}$ feature (Hrivnak et al. 2007). New mid-IR spectra have also been obtained of six carbon-rich PPNe using Spitzer. These reveal one new $21 \mu \mathrm{m}$ source and give good observations of the others. Also seen are the 11.3 and $12.3 \mu \mathrm{m}$ emission bands. 
Table 1. Summary of the Spectral Features of Carbon-Rich PPNe and $21 \mu \mathrm{m}$ Sources $^{a}$

\begin{tabular}{|c|c|c|c|c|c|c|c|c|c|c|c|c|c|c|c|c|c|}
\hline Object & SpT & $\mathrm{C} / \mathrm{O}$ & $\mathrm{C}_{2}, \mathrm{C}_{3}$ & 3.3 & 3.4 & 6.2 & 6.9 & 7.7 & 8.6 & $8 \mathrm{br}$ & 11.3 & 12.3 & Class $^{b}$ & $\mathrm{C}_{2} \mathrm{H}_{2}$ & 15.8 & 21 & $30 \mu \mathrm{m}$ \\
\hline $02229+6208$ & G8 Ia & $\ldots$ & $\mathrm{Y}, \mathrm{Y}$ & Y & $Y:^{*}$ & $\mathrm{Y}:$ & $\mathrm{Y}$ & $\mathrm{N}$ & $\mathrm{N}$ & $\mathrm{Y}$ & $\mathrm{Y}$ & $\mathrm{Y}$ & A & $\ldots$ & & Y & $\mathrm{Y}$ \\
\hline $20000+3239$ & G8 Ia & $\ldots$ & $\mathrm{Y}, \ldots$ & Y & $\mathrm{Y}^{*}$ & $\mathrm{Y}$ & Y & $\mathrm{N}$ & $\mathrm{N}$ & Y & Y & Y & A & $\ldots$ & & Y & $\mathrm{Y}$ \\
\hline $05113+1347$ & G8 Ia & 2.4 & $\mathrm{Y}, \mathrm{Y}$ & $\mathrm{Y}:$ & Y: & & & & & & Y & $\mathrm{Y}$ & & $\mathrm{N}:^{*}$ & $Y:^{*}$ & Y & $\mathrm{Y}$ \\
\hline $22272+5435$ & G5 Ia & 1.6 & $\mathrm{Y}, \mathrm{Y}$ & $\mathrm{Y}$ & Y & $\mathrm{Y}$ & $\mathrm{Y}$ & $\mathrm{Y}$ & $\mathrm{N}$ & Y & Y & $\mathrm{Y}$ & B & & $\mathrm{Y}:$ & $\mathrm{Y}$ & $\mathrm{Y}$ \\
\hline $07430+1115$ & G5 Ia & $\ldots$ & $\mathrm{Y}, \mathrm{Y}$ & $\mathrm{Y}$ & $\mathrm{Y}$ & $\ldots$ & $\ldots$ & $\ldots$ & $\ldots$ & Y: & Y: & $\ldots$ & A & $\ldots$ & $\ldots$ & $\mathrm{Y}^{*}$ & Y \\
\hline $23304+6147$ & G2 Ia & 2.8 & $\mathrm{Y}, \mathrm{Y}$ & $\mathrm{Y}^{*}$ & $Y:^{*}$ & $\mathrm{Y}$ & $\mathrm{Y}$ & $\mathrm{Y}$ & $\mathrm{Y}$ & $\mathrm{Y}$ & $\mathrm{Y}$ & $\mathrm{Y}$ & A & $Y:^{*}$ & $\mathrm{Y}^{*}$ & $\mathrm{Y}$ & $\mathrm{Y}$ \\
\hline $05341+0852$ & G2 Ia & 1.6 & $\mathrm{Y}, \mathrm{Y}$ & $\mathrm{Y}$ & $\mathrm{Y}$ & $\mathrm{Y}$ & $\mathrm{Y}$ & $\mathrm{N}$ & $\mathrm{N}$ & $\mathrm{Y}$ & $\mathrm{Y}$ & $\mathrm{Y}^{*}$ & B & $\mathrm{Y}^{*}$ & $\mathrm{Y}^{*}$ & $\mathrm{Y}$ & $\mathrm{Y}$ \\
\hline $22223+4327$ & G0 Ia & 1.2 & $\mathrm{Y}, \mathrm{Y}$ & Y & $\mathrm{N}$ & & & & & & Y & & $\mathrm{A}$ & & & $\mathrm{Y}$ & $\mathrm{Y}$ \\
\hline $04296+3429$ & G0 Ia & $\ldots$ & $\mathrm{Y}, \mathrm{Y}$ & $\mathrm{Y}$ & $\mathrm{Y}$ & & & $\mathrm{Y}$ & & & $\mathrm{Y}$ & $\mathrm{Y}$ & B & & & $\mathrm{Y}$ & $\mathrm{Y}$ \\
\hline AFGL 2688 & F5 Iae & 1.0 & $\mathrm{Y}, \mathrm{Y}$ & $\mathrm{Y}$ & Y & Y & $\mathrm{Y}:$ & $\mathrm{N}$ & $\mathrm{N}$ & $\mathrm{Y}$ & Y & $\mathrm{N}:$ & A & Y & & Y: & Y \\
\hline $06530-0230$ & F5 I & 2.8 & $\mathrm{Y}, \mathrm{Y}$ & $\mathrm{Y}^{*}$ & $\mathrm{~N}$ & $\ldots$ & $\ldots$ & $\ldots$ & $\ldots$ & 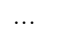 & $\mathrm{Y}^{*}$ & $\mathrm{Y}^{*}$ & A & $\mathrm{Y}^{*}$ & $\mathrm{Y}^{*}$ & $\mathrm{Y}^{*}$ & $\mathrm{Y}^{*}$ \\
\hline $07134+1005$ & F5 I & 1.0 & $\mathrm{Y}, \mathrm{N}$ & $\mathrm{Y}$ & Y: & $\ldots$ & $\mathrm{Y}$ & $\mathrm{Y}$ & $\mathrm{N}$ & $\mathrm{Y}$ & $\mathrm{Y}$ & $\mathrm{Y}$ & A & & Y: & $\mathrm{Y}$ & $\mathrm{Y}$ \\
\hline $19500-1709$ & F3 I & 1.0 & $\mathrm{~N}, \mathrm{~N}$ & $\mathrm{~N}$ & $\mathrm{~N}$ & & & & & $\mathrm{Y}:$ & $\mathrm{Y}$ & Y: & $\ldots$ & & Y: & $\mathrm{Y}$ & $\mathrm{Y}$ \\
\hline $16594-4656$ & B7 & $\ldots$ & $\mathrm{N}, \mathrm{N}$ & $\mathrm{Y}$ & $\mathrm{N}$ & $\mathrm{Y}$ & $\mathrm{N}$ & $\mathrm{Y}$ & $\mathrm{Y}$ & Y: & $\mathrm{Y}$ & Y: & A & & & $\mathrm{Y}$ & $\mathrm{Y}$ \\
\hline $01005+7910$ & B0 I & 1.2 & $\mathrm{~N}, \mathrm{~N}$ & $\mathrm{Y}$ & $\mathrm{Y}^{*}$ & $\mathrm{Y}$ & $\mathrm{N}$ & $\mathrm{Y}$ & $\mathrm{Y}$ & $\mathrm{N}$ & $\mathrm{Y}$ & $\mathrm{N}$ & A & & & $\mathrm{N}:$ & $\mathrm{Y}$ \\
\hline $22574+6609$ & $\ldots$ & $\ldots$ & $\ldots, \ldots$ & $\ldots$ & $\ldots$ & $\mathrm{Y}$ & $\mathrm{Y}$ & $\mathrm{Y}$ & $\mathrm{N}$ & $\mathrm{Y}$ & $\mathrm{Y}$ & $\mathrm{Y}^{*}$ & $\ldots$ & $\mathrm{Y}^{*}$ & $\mathrm{~N}^{*}$ & Y & $\mathrm{Y}$ \\
\hline $19477+2401$ & $\ldots$ & $\ldots$ & $\ldots, \ldots$ & $\ldots$ & $\ldots$ & $\ldots$ & $\ldots$ & $\ldots$ & $\ldots$ & $\ldots$ & $\ldots$ & $\ldots$ & $\ldots$ & $\ldots$ & $\ldots$ & $\mathrm{Y}^{*}$ & $\mathrm{Y}$ \\
\hline
\end{tabular}

Note 1: Colon indicates a marginal or uncertain detection, blank indicates lack of information, "..." indicates that the object has not been observed in this spectral region.

Note 2: Asterisk indicates a new detection from Hrivnak et al. (2007) or Hrivnak et al. (2008).

${ }^{a}$ Table does not include three newly discovered C-rich PPNe IRAS 08143-4406, 08281-4850, 14325-6428 (Reyniers et al. 2004, 2007) that have not been observed in the IR.

${ }^{b}$ Classification scheme of Geballe (1997) at 3.3, 3.4 $\mu \mathrm{m}$.

Two other emission features are seen. At $15.8 \mu \mathrm{m}$ is a new, relatively strong, unidentified feature seen in four sources; it is strongest in the two with the strongest $21 \mu \mathrm{m}$ feature. At $13.7 \mu \mathrm{m}$ is seen the $\mathrm{C}_{2} \mathrm{H}_{2}$ feature in four sources, including the first report of $\mathrm{C}_{2} \mathrm{H}_{2}$ in emission in a post-AGB object (Hrivnak et al. 2008). Results are listed in Table 1.

\section{Summary}

- 3.3, $3.4 \mu \mathrm{m}$ : All C-rich PPNs have $3.3 \mu \mathrm{m}$ and most have $3.4 \mu \mathrm{m}$ emission features.

- $21 \mu \mathrm{m}$ : (a) All have the same shape and central wavelength $(20.1 \pm 0.1 \mu \mathrm{m})$ but differ in strength; (b) all are C-rich, (almost) all show $\mathrm{C}_{2}, \mathrm{C}_{3}, 3.3,11.3,30 \mu \mathrm{m}$ emission.

- $\mathrm{C}_{2} \mathrm{H}_{2}$ : (a) Detected in four $21 \mu \mathrm{m}$ sources; all show P-Cygni profiles; (b) first detection in emission in post-AGB stars.

- $15.8 \mu \mathrm{m}$ : New feature seen in several of the PPNe including previous ISO spectra; unidentified; (b) correlated with $21 \mu \mathrm{m}$ emission?

- Trends: (a) All $21 \mu \mathrm{m}$ sources are C-rich, (almost) all show $\mathrm{C}_{2}, \mathrm{C}_{3}, 3.3,11.3,30 \mu \mathrm{m}$ emission; (b) no correlation found between $3.4 / 3.3$ ratio and spectral type.

\section{Acknowledgements}

BJH acknowledges support from NASA (JPL/Caltech 1276197) and NSF (AST-0407087).

\section{References}

Geballe, T. R. 1997, in: Y. J. Pendleton \& A. G. G. M. Tielens (eds.), From Stardust to Planetesimals, (ASP: San Francisco), p. 119

Geballe, T. R., Tielens, A. G. G. M., Kwok, S., \& Hrivnak, B. J. 1992, ApJ (Letters), 387, L89

Hrivnak, B. J., Geballe, T. R., \& Kwok, S. 2007, ApJ, 662, 1059

Hrivnak, B. J., Volk, K., \& Kwok, S. 2008, ApJ, submitted

Kwok, S. 2004, Nature, 430, 985

Kwok, S., Volk, K., \& Hrivnak, B. J. 1989, ApJ (Letters), 345, L51

Reyniers, M., van Winckel, H., Gallino, R., \& Straniero, O. 2004, A\&A, 417, 269

Reyniers, M., Van de Steene, G. C., van Hoof, P. A. M., \& van Winckel, H. 2007, A\&A, 471,247

Speck, A. K. \& Hofmeister, A. M. 2004, ApJ, 600, 986

Volk, K., Kwok, S., \& Hrivnak, B. J. 1999, ApJ (Letters), 516, L99 